\section{Frühgeburt nimmt Haut in Schutz}

\section{Frühchen mit einem extrem niedrigen Geburtsgewicht sind durch die Unreife von Haut und Schleimhaut in verstärktem Maße schutzlos zahlreichen Antigenen ausgesetzt. Das Erstaunliche: Ein Jahr nach ihrer Geburt zeigen sie eine signifikant geringere Neigung zur Ausbildung eines atopischen Ekzems als normalgewichtig geborene Kinder.}

Frühgeburten sind im Allgemeinen sehr krankheitsanfällig. Als Hochrisikokinder wurden sie auch hinsichtlich einer Sensibilisierung gegen zahlreiche Antigene eingestuft. Denn die Haut wie auch die Schleimhäute von Gastrointestinaltrakt und Bronchialsystem sind noch unreif und lassen möglicherweise vermehrt Antigene passieren. Ein gehäuftes Zusammentreffen von Geburt vor dem errechneten Termin und beispielsweise Neurodermitis im ersten Lebensjahr ließ sich aber epidemiologisch nie nachweisen.

\section{Geringe Neurodermititisrate \\ 1 Jahr nach Geburt}

Möglicherweise liegt sogar ein inverser Zusammenhang vor, wie jetzt eine Arbeitsgruppe vom Virchow-Klinikum in Berlin mitteilte. In die Studie waren 315 Frühchen mit extrem niedrigem Geburtsgewicht von unter $1500 \mathrm{~g}$ einbezogen, die man ein Jahr nach ihrer ter auf das Vorliegen eines atopischen Ekzems (AE) hin untersuchen ließ. Gleichzeitig wurden die Angaben über familiäre Atopiebelastung, sonstige dermatologische Beobachtungen durch die Eltern sowie Rauchgewohnheiten und Einkommen der Eltern erfragt. Die so erhobenen Daten verglich man mit denen von 455 Kontrollkindern, die mit Normalgewicht geboren worden waren.

Nur 2\% der Frühgeborenen hatten zum Untersuchungszeitpunkt ein atopisches Ekzem, während dies auf 4\% der Kontrollkinder zutraf. In dieser Gruppe berichteten einige Eltern auch, Geburt durch einen geschulten Pädia- daß es bei den Kindern in den vergangenen 12 Monaten einige Male $\mathrm{zu}$ vorübergehenden Hautsymptomen gekommen war.

\section{Antigenspezifische TH2-Zellen werden supprimiert}

Die Prävalenz des atopischen Ekzems zum Zeitpunkt der Untersuchung wie auch die kalkulierte Wahrscheinlichkeit, im ersten Lebensjahr ein $\mathrm{AE}$ zu entwickeln ( $1,5 \%$ vs. $4,6 \%)$ war in der Gruppe der Frühgeborenen mit geringem Geburtsgewicht signifikant niedriger als bei den normalgewichtigen Kindern. Zigarettenkonsum und Einkommen der Eltern hatten keinen Einfluß auf die Haut der Kleinen.

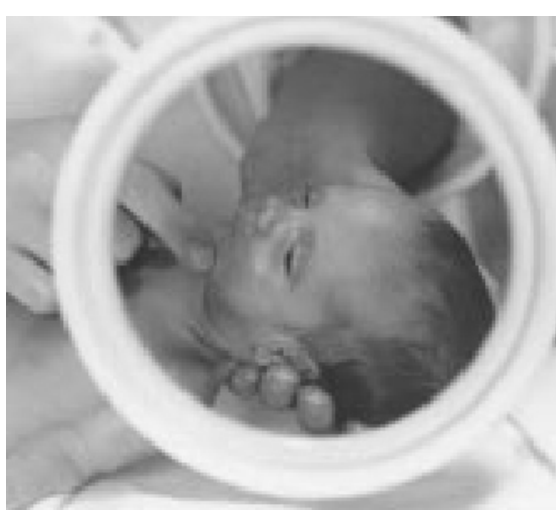

Frühgeborene - durch Konfrontation mit hohen Allergenmengen vor Neurodermitis geschützt?

Eine schlüssige Erklärung für diese Beobachtung, so die Autoren, könne man noch nicht anbieten. Die frühe Konfrontation einer physiologisch noch nicht reifen Haut mit Allergenen scheint aber tatsächlich eher zur Ausbildung einer Toleranz als $\mathrm{zu}$ einer Sensibilisierung der Kinder beizutragen. Aus Versuchen mit Mäusen weiß man, daß durch die Verfütterung kleiner Antigenmengen die antigenspezifischen TH2-Lymphozyten und die IgEProduktion unterdrückt werden. Möglicherweise liegt hier ein Erklärungsansatz.

Bührer Ch, Grimmer I, Niggemann B. Low 1-year prevalence of atopic eczema in very low birthweight infants. Lancet 1999 ;
353: 1674 .

\section{Asthma exazerbiert: Oft stecken Viren dahinter}

Die Erkältungs- und Grippesaison hat begonnen. Virale Atemwegsinfekte gefährden nun wie jedes Jahr wieder die Asthmatiker - denn unter einem viralen Infekt exazerbiert die Asthmasymptomatik besonders oft.

2 amerikanische Untersuchungen belegen dies erneut: In einer Longitudinalstudie wurden 29 stationär behandelte Asthmapatienten 30 Monate lang beobachtet. An 44\% der in dieser Zeit aufgetretenen Asthmaexazerbationen waren Viren beteiligt, 2 von 3 Atemwegsinfekten gingen mit einer Verschlechterung der Symptome in den unteren Atemwegen einher.

In einer Längsschnittstudie an der selben Klinik untersuchte man 129 erwachsene Patienten, die sich wegen akuter Verschlechterung ihrer Asthmasymptomatik in der Notfallambulanz eingefunden hatten. Hier wurden die Ärzte bei 55\% der Patienten fündig: Die Asthmatiker litten gleichzeitig unter Atemwegsinfekten, hervorgerufen durch Rhino- oder Influenzaviren. $b k$ 